\title{
Lossless Compression of Pre-press Images Using a Novel Colour Decorrelation Technique
}

\author{
Steven Van Assche, Wilfried Philips, and Ignace Lemahieu \\ Department of Electronics and Information Systems, University of Ghent \\ St.-Pietersnieuwstraat 41, B-9000 Gent, Belgium \\ E-mail: svassche@elis.rug.ac.be
}

\begin{abstract}
In the pre-press industry colour images have both a high spatial and a high colour resolution. Such images require a considerable amount of storage space and impose long transmission times. Data compression is desired to reduce these storage and transmission problems. Most existing compression schemes operate on gray-scale images. However, in the case of colour images higher compression ratios can be achieved by exploiting inter-colour redundancies.

In this paper a new lossless colour transform is proposed, based on the KLT. This transform removes redundancies in the colour representation of each pixel and can be combined with many existing compression schemes. In this paper it is combined with a prediction scheme that exploits spatial redundancies.

The results proposed in this paper show that the colour transform typically saves about a half to two bit per pixel, compared to a purely predictive scheme. The results also suggest that combining the proposed KLT scheme with the state-of-the-art CALIC gray-scale-only coder could significantly increase the compression ratio of that scheme.
\end{abstract}

\section{Introduction}

In pre-press companies documents (e.g., magazines, brochures and advertising posters) are usually composed electronically nowadays. These documents contain images with high spatial resolution (typically, $2000 \times 2000$ pixels) and high colour resolution ( 24 bit per pixel for RGB-images and 32 bit per pixel for CMYK-images, which are more commonly used). Hence, such images occupy considerable amounts of storage space (typically $16 \mathrm{Mbyte} / \mathrm{image}$ ), and also pose transmission problems (e.g., transmitting images from a pre-press house to a printing company over ISDN-lines takes 15 to 30 minutes).

Clearly, data compression can significantly alleviate there problems. Because of the high quality requirements imposed by the customers, the pre-press industry is reluctant to adopt lossy compression techniques.

As far as lossless compression is concerned, many techniques are available, at least for gray-scale images. Some of the current state-of-the-art lossless compression techniques for contone gray-scale images are lossless JPEG [12] (the current standard), BTPC [9] (a binary piramid coder), FELICS [5] (a "fast efficient lossless image coder"), the S+P-transform [11,10,3] (an integer wavelet coder) and 
CALIC [13] (a highly optimized technique with a complex prediction scheme). According to our experiments [1], CALIC yields the highest lossless compression rates (of the order of 2), but it is also the slowest of the techniques mentioned.

In the case of colour images, the above techniques usually process each of the colour components independently, i.e., the gray-scale images obtained after colour separation are encoded separately. In this case, the K-component of a CMYK-image usually compresses better than the other components (factor of 3 to 4 instead of 2), but the mean compression ratio for all components remains of the order of 2 . It is clear that higher compression ratios can be achieved by exploiting inter-component redundancies. For instance, it is well-known that for CMYK-images the K-component is highly correlated with the three other components.

Nowadays, only few proposed techniques exploit such colour redundancies. Two noteworthy exceptions operate on RGB colour images [6,2]. Both techniques first compress the red component and then use information derived from the red component to predict the green component; subsequently, the green component is used in the same manner to predict the blue component. Unfortunately, this approach provides only a slight improvement in compression ratio (about 5\%).

This paper proposes a new technique for exploiting inter-component redundancies. The technique is based on a modified Karhunen-Loève transform (KLT) scheme in combination with a novel quantization scheme that guarantees losslessness. The KLT decorrelates the colour components. It is recomputed on a block by block basis and is therefore spatially adaptive. In the current implementation spatial redundancies are removed using predictive techniques (mainly lossless JPEG) to facilitate an easy comparison with pure gray-scale techniques.

Section 2 describes how the modified KLT is able to achieve both a lossless transform of the image components and a good compression ratio, which the usual KLT, and in fact all floating point transforms, are uncapable of (when they achieve losslessness, the compression ratio drops to about 1 ). Section 3 gives an overview of the complete compression scheme. Section 4 presents experimental results on many pre-press images. These results show that an increase of more than $10 \%$ in compression ratio is possible over pure gray-scale techniques.

\section{Lossless Transform Coding}

In lossy transform coding a data vector $\mathbf{d}$, consisting of sample values of a signal or image, is multiplied by an orthogonal transform matrix $T$, which results in a coefficient vector $\mathbf{a}=T \mathbf{d}$. In the following we assume that the components of $\mathbf{d}$ are integers. A "good" transform yields coefficients $a_{i}$ which are very little correlated. This is important because the rate-distortion theory predicts that the rate-distortion bound can be reached by coding each of the coefficients independently of the others, which greatly simplifies the coding problem. In practical transform coders, the coefficients $a_{j}$ are quantized, i.e., converted into integers $a_{j}^{\prime}$, which are then entropy-coded. In the simplest case (uniform quantization), $a_{j}^{\prime}=\left[a_{j} / \Delta\right]$, where $[x]$ denotes the integer nearest to $x$. In lossy compression, $\Delta$ 
is used to control the compression ratio (e.g., increasing $\Delta$ causes a decrease in bit rate at the expense of larger coding errors). Decompression involves transforming the quantized coefficients into an approximation $\mathrm{d}^{\prime}=\left[\Delta T^{t} \mathbf{a}^{\prime}\right]$ of the original data vector $\mathbf{d}$.

In principle, all transform coding techniques can be made lossless by sufficiently reducing the quantization step $\Delta$. Indeed, let $N$ be the dimension of $\mathbf{d}$. It is easily shown that $\mathbf{d}^{\prime}=\mathbf{d}$ for all possible data vectors $\mathbf{d}$ when $\Delta \leq 1 / \sqrt{N}$; this is because $\left\|\mathbf{d}^{\prime}-\mathbf{d}\right\|=\left\|\Delta \mathbf{a}^{\prime}-\mathbf{a}\right\|$ and because $\left|\Delta a_{j}^{\prime}-a_{j}\right|<\Delta / 2$, which implies that $\left|d_{j}^{\prime}-d_{j}\right| \leq\left\|\mathbf{d}^{\prime}-\mathbf{d}\right\|<\sqrt{N} \Delta / 2<1 / 2$. It can also be shown that in general, a transform coding technique cannot be lossless when $\Delta>1 / \sqrt{N}$. Unfortunately, the compression ratio that corresponds to the minimum value of $\Delta$ is usually lower than 1 , which means that lossless transform coding is useless in practice, at least in the manner described.

In principle, transform coding techniques can also be made lossless by using higher values of $\Delta$ and coding the residual error. However, this approach does not work either, because the residual image is noise-like and cannot be compressed well. We now present a novel technique for turning a lossy transform coding scheme into a successful lossless scheme. The procedure is based on the fact that any matrix $T$ (even a non-orthogonal one) can be well approximated by two prediction operators $P_{1}[$.$] and P_{2}[$.$] which map integers into integers, followed$ by a floating point scaling step:

$$
\mathbf{a}=T \mathbf{d} \approx D P_{2}\left[P_{1}[\mathbf{d}]\right]
$$

where $D$ is a diagonal matrix.

An important property of the prediction operators $P_{1}[$.$] and P_{2}[$.$] is that$ they are invertible when applied to integer vectors. Now consider the transform $\mathbf{a}^{\prime \prime}=P[\mathbf{d}]$, where $P[\mathbf{d}]=P_{2}\left[P_{1}[\mathbf{d}]\right]$. This operator maps integer data vectors $\mathbf{d}$ into integer coefficient vectors $\mathbf{a}^{\prime \prime}$ and is lossless. Furthermore, in view of eq. (1), $\mathbf{a}^{\prime \prime} \approx D^{-1} \mathbf{a}^{\prime}$. Therefore, the statistical properties of the coefficients $a_{j}^{\prime \prime}$ are similar to those of the corresponding coefficients $a_{j}^{\prime}$ in an "ordinary" transform scheme, but with a quantization step $\Delta_{j}=1 / D_{j, j}$, which differs from coefficient to coefficient. Note that simply quantizing the coefficients $a_{j}$ using the quantization steps $\Delta_{j}$, does not produce quantized coefficients $a_{j}^{\prime}$ from which the integer data vector $\mathbf{d}$ can be error-freely recovered.

\section{An overview of the lossless compression scheme}

Figure 1 displays an overview of the proposed coder and Fig. 2 of the corresponding decoder. In the following paragraphs we briefly describe the different steps of the coding process. We will not discuss the decoder in detail because it is basically the inverse of the coder, but we will point out the differences where appropriate.

In the proposed scheme, spatial redundancies are removed using a losslessJPEG-like prediction scheme, while colour redundancy is removed using the 


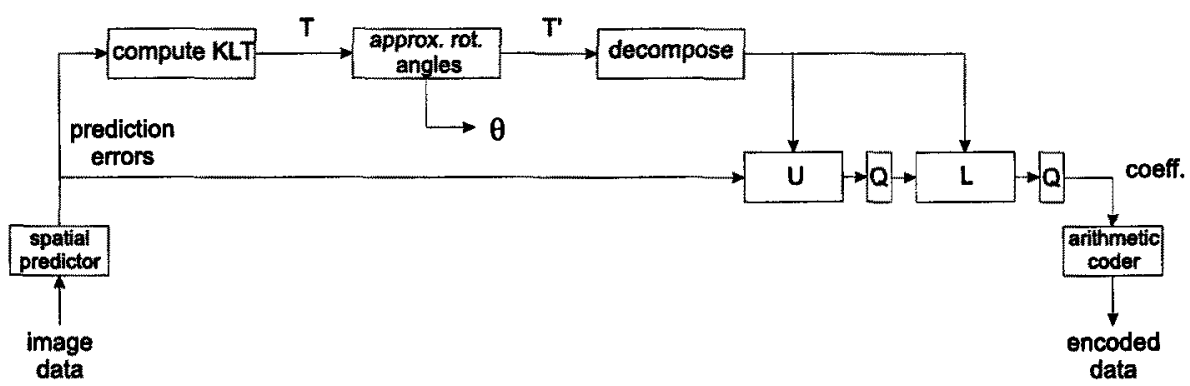

Fig. 1: The encoder of the proposed scheme

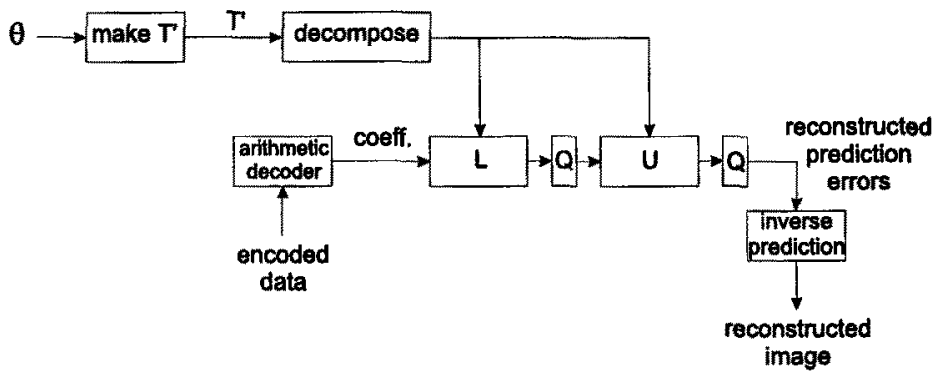

Fig. 2: The decoder of the proposed scheme

lossless KLT (the KLT may be applied before or after the prediction step). Finally, the resulting coefficients are presented to an arithmetic coder.

\subsection{The lossless Karhunen-Loève transform}

The Karhunen-Loève transform [8] is defined as the transform which decorrelates the components of a random vector. In classical compression schemes decorrelation is usually applied in the spatial domain and is usually replaced by the DCT which is close to optimal in practice. In any case, the KLT can be wellapproximated by a lossless operator, as explained in the previous section. We will refer to this operator as the lossless KLT.

In the proposed scheme the lossless KLT is applied in the colour domain, i.e., to transform the colour values of a pixel into decorrelated numbers. In fact the KLT is computed for a block of pixels and is therefore block-dependent. This is to exploit the fact that the colour statistics in an image can vary strongly from region to region.

As the lossless KLT for a block depends on the colour statistics in that block, which are unknown to the receiver, side information must be transmitted so that the receiver can reconstruct the lossless KLT itself. For this purpose, we use the following scheme: we start by computing the orthogonal KLT matrix $T$ for a 
given block; next, we expand $T$ as the product of rotation matrices, which are each completely specified by one rotation angle. These rotation angles are quantized to 8 bit, and are used to construct an (exactly orthogonal) approximation $T^{\prime}$ of $T$. The lossless KLT is then derived from $T^{\prime}$ (instead of from $T$ ).

The quantized rotation angles are sent to the receiver which from them reconstructs the lossless KLT. The involved overhead is negligible in practice because each rotation angle requires only 8 bit and because the number of rotation angles is small (i.e., 3 for $N=3$ and 6 for $N=4$ ).

\subsection{Spatial prediction and arithmetic coding}

In principle, any spatial decorrelation scheme may be combined with the above lossless KLT colour transform to further remove (spatial) redundancies. In the current implementation, we use a simple linear predictor, i.e., predictor 7 as described in the Lossless JPEG-standard. If $I_{a}$ and $I_{l}$ are the intensities of the pixel above and the pixel to the left of the pixel to be coded, then the prediction is $\left\lfloor\left(I_{a}+I_{l}\right) / 2\right\rfloor$, where $\lfloor x\rfloor$ is the largest integer not greater than $x$.

The data which remains after the (spatial and colour) decorrelation is very non-uniformly distributed. Therefore, it should be entropy-coded. In principle, both Huffman and arithmetic coders can be used. However, as arithmetic coders can easily adapt to varying data statistics and therefore usually produce higher data compressions, we have opted for an arithmetic coder in the proposed scheme. Specifically, we used the the "bit-plus-follow" version of the coder described in [4]. In the current implementation, the arithmetic coder is reinitialized in each block using some block-dependent information, which is also sent to the receiver.

\section{Experimental Results}

We have implemented the proposed scheme and tested it on several (mainly prepress) RGB- and CMYK-images. In each case we reconstructed the image and verified the losslessness of the proposed scheme.

In order to evaluate the compression gain due to the lossless KLT colour processing step, and to compare it to the gain due the spatial decorrelation step, we first compressed one image (the 32 bit CMYK "musicians" image which is representative for pre-press applications) with and without spatial prediction and with and without the lossless KLT. The same block size is used in both the prediction and the colour decorrelation step.

Table 1 shows that even without spatial prediction the KLT achieves a bit rate which is not much higher than that of a purely spatial predictive scheme. In combination with spatial prediction it yields an even lower bit rate. Table 1 also illustrates that it is best to apply the lossless KLT step after the spatial prediction step, as a kind of error modeling.

Figure 3 depicts the influence of the block size for the same image. As expected, for the purely predictive technique large blocks yield a better compression, because the arithmetic coder is then able to build up more reliable statistics. 
Table 1: Bit rate (bpp) for musicians with block size $30 \times 30$ pixels

\begin{tabular}{l|c|c|c|c|c|c|} 
& Number of pixels & Original & $\begin{array}{c}\text { Prediction } \\
\text { only }\end{array}$ & $\begin{array}{c}\text { KLT } \\
\text { only }\end{array}$ & $\begin{array}{c}\text { Prediction } \\
\text { after KLT }\end{array}$ & $\begin{array}{c}\text { Prediction } \\
\text { before KLT }\end{array}$ \\
\hline musicians & $1853 \times 2103$ & 32 & 19.10 & 19.86 & 17.84 & 16.67
\end{tabular}

On the other hand, the results show that the colour decorrelation is more efficient at smaller block sizes. For "musicians", the optimal compromise is a block size of $30 \times 30$. Our experiments have shown that the optimal block size strongly depends on the image (optimal block sizes vary from $10 \times 10$ to $100 \times 100$ ); however, the bit rate does not vary rapidly as a function of the block size.

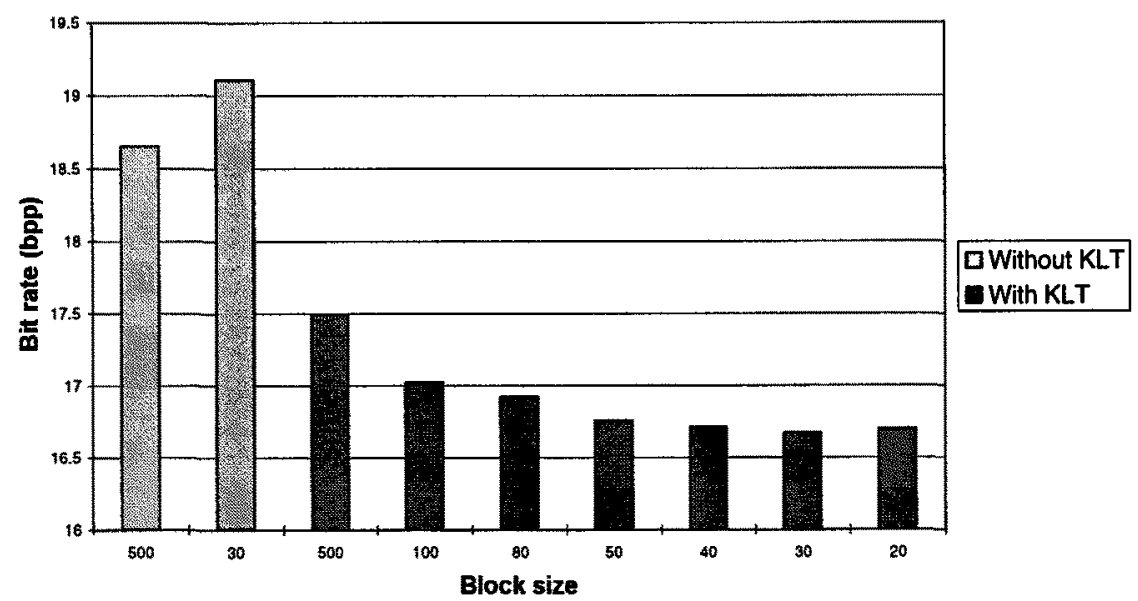

Fig. 3: Influence of the block size on the compression of musicians

Table 2 shows compression results for some CMYK and RGB colour images, including the well-known "lena" and "peppers" images. In each case, the bit rate is listed for three different schemes: the spatial-prediction-only scheme, the proposed scheme (spatial prediction plus KLT) and the CALIC scheme (which is applied after colour separation).

The results in table 2 show that the KLT-step leads to a considerable decrease in bit rate ( 0.5 to 2 bit per pixel) on most images. One exception is the image tools, which is not really representative and which also poses problems to other compression algorithms (e.g., see the bit rate for CALIC).

Table 2 also shows that the CALIC scheme always outperforms the proposed scheme, even though it does not exploit colour redundancy. The results suggest that combining the KLT-colour process step with CALIC would enable an additional reduction in bit rate over the CALIC results. Unfortunately, exper- 
Table 2: Bit rate (bpp) for some colour images

\begin{tabular}{c|c|cccc} 
& & \multicolumn{4}{c}{ Without With } \\
& Number of pixels & Original & KLT & KLT & CALIC \\
\hline musicians & $1853 \times 2103$ & 32 & 19.10 & 16.67 & 16.58 \\
scid0 & $2048 \times 2560$ & 32 & 16.00 & 14.95 & 14.16 \\
scid3 & $2048 \times 2560$ & 32 & 17.02 & 15.84 & 13.85 \\
koekjes1 & $827 \times 591$ & 32 & 18.71 & 18.08 & 16.00 \\
koekjes2 & $839 \times 638$ & 32 & 17.88 & 17.49 & 15.76 \\
timep_xfld & $339 \times 432$ & 32 & 18.71 & 19.05 & 16.58 \\
tools $^{\dagger}$ & $1524 \times 1200$ & 32 & 23.36 & 23.53 & 19.75 \\
woman $^{\dagger}$ & $2048 \times 2560$ & 32 & 17.78 & 16.33 & 16.16 \\
bike $^{\dagger}$ & $2048 \times 2056$ & 32 & 16.67 & 15.31 & 14.04 \\
cafe $^{\dagger}$ & $2048 \times 2056$ & 32 & 22.38 & 21.48 & 18.82 \\
\hline \hline water $^{\dagger}$ & $3072 \times 2048$ & 24 & 5.56 & 5.14 & 5.21 \\
cats $^{\dagger}$ & $3072 \times 2048$ & 24 & 8.24 & 7.66 & 7.54 \\
cmpnd2 $^{\dagger}$ & $1024 \times 1400$ & 24 & 5.66 & 5.59 & 3.72 \\
graphic $^{\dagger \dagger}$ & $2644 \times 3046$ & 24 & 8.35 & 7.88 & 6.78 \\
chart_s $^{\dagger}$ & $1688 \times 2347$ & 24 & 11.30 & 10.97 & 7.99 \\
lena & $512 \times 512$ & 24 & 15.00 & 14.20 & 13.19 \\
peppers $^{\dagger}$ & $512 \times 512$ & 24 & 15.79 & 15.48 & 13.87
\end{tabular}

iments have shown that applying CALIC after colour decorrelation does not yield any compression gain. This is not surprising because the statistics of the decorrelated data differs significantly from the statistics of the original data and because CALIC was not designed for such data. However, according to [13], the spatial prediction step accounts for most of the data reduction in CALIC and this predictor can easily be combined with our colour decorrelation scheme. Unfortunately, we have not been able to merge our scheme with the CALIC predictor because no source code is available for CALIC.

\section{Conclusion}

This paper introduces a new lossless colour transform, based on the KLT. The transform removes redundancies in the colour representation of each pixel but not inter-pixel redundancies and is therefore combined with a prediction scheme that exploits spatial redundancies.

The proposed scheme is tested on several images and the results show that it typically saves about a half to two bit per pixel, compared to a purely predictive scheme. The results also suggest that combining the proposed KLT scheme with the state-of-the-art CALIC gray-scale-only coder could significantly increase the compression ratio of that scheme.

\footnotetext{
${ }^{\dagger}$ Found at ftp://ipl.rpi.edu/pub/image2/jpeg_cont_tone/.

${ }^{\ddagger}$ Graphics is an image represented in the $1^{*} a^{*} b^{*}$ colour-space.
} 


\section{Acknowledgments}

This work was financially supported by the Belgian National Fund for Scientific Research (NFWO) through a mandate of "postdoctoral research fellow" and through the projects 39.0051 .93 and 31.5831 .95 , and by the Flemish Institute for the Advancement of Scientific-Technological Research in Industry (IWT) trough the projects Tele-Visie (IWT 950202) and Samset (IWT 950204).

\section{References}

1. K. Denecker, J. Van Overloop, and I. Lemahieu. An experimental comparison of several lossless image coders for medical images. In S. Hagerty and R. Renner, editors, Proceedings of the Data Compression Industry Workshop, pages 67-76, Snowbird, Utah, USA, March 1997. Ball Aerospace \& Technologies Corp.

2. Koen Denecker and Ignace Lemahieu. Lossless colour image compression using inter-colour error prediction. In J.-P. Veen, editor, Proceedings of the PRORISC IEEE Benelux Workshop on Circuits, Systems and Signal Processing, pages 95100, Mierlo, Nederland, November 1996. STW Technology Foundation.

3. Steven Dewitte and Jan Cornelis. Lossless integer wavelet transform. IEEE Signal Processing Letters, 1997. To be published.

4. Paul G. Howard and Jeffrey Scott Vitter. Arithmetic coding for data compression. Proceedings of the IEEE, 82(6):857-865, June 1994.

5. Paul Glor Howard. The Design and Analysis of Efficient Lossless Data Compression Systems. PhD thesis, Department of Computer Science, Brown University, Providence, Rhode Island, June 1993.

6. Nasir D. Memon and Khalid Sayood. Lossless compression of RGB color images. Optical Engineering, 34(6):1711-1717, 1995.

7. W. Philips and K. Denecker. A new embedded lossless/quasi-lossless image coder based on the Hadamard transform. In Proceedings of the IEEE International Conference on Image Processing (ICIP97), 1996. To be published.

8. William K. Pratt. Digital image processing. New-York: Wiley-Interscience, second edition, 1991.

9. John A. Robinson. Efficient general-purpose image compression with binary tree predictive coding. IEEE Transactions on Image Processing, 6(4):601-608, April 1997.

10. Amir Said and William A. Pearlman. Image compression via multiresolution representation and predictive coding. In Visual Communications and Image Processing, number 2094 in SPIE, pages 664-674, November 1993.

11. Amir Said and William A. Pearlman. An image multiresolution representation for lossless and lossy compression. In SPIE Symposium on Visual Communications and Image Processing, Cambridge, MA, November 1993.

12. The International Telegraph and Telephone Consultative Committee (CCITT), editors. Digital Compression and Coding of Continuous-Tone Still Images. Recommendation T.81, 1992.

13. Xiaolin Wu and Nasir Memon. CALIC - a context based adaptive lossless image codec. In IEEE International Conference on Acoustics, Speech, \& Signal Pocessing, volume 4, pages 1890-1893, May 1996. 